\title{
Estimation of Ice Sheet Attenuation by Using Radar Sounder and Ice Core Data
}

\author{
Ana-Maria Ilisei ${ }^{\mathrm{a}}, \mathrm{Jilu} \mathrm{Li}^{\mathrm{b}}$, Sivaprasad Gogineni ${ }^{\mathrm{b}}$, and Lorenzo Bruzzone ${ }^{\mathrm{a}}$ \\ ${ }^{a}$ University of Trento, Dept. Information Engineering and Computer Science, Via Sommarive \\ 5, I-38123, Trento, Italy, anamaria.ilisei@unitn.it \\ ${ }^{\mathrm{b}}$ Center for Remote Sensing of Ice Sheets, The University of Kansas, Nichols Hall 2335 Irving \\ Hill Road, Lawrence, Kansas 66045, USA.
}

\begin{abstract}
Due to their great impact on the environment and society, the study of the ice sheets has become a major concern of the scientific community. In particular, the estimation of the ice attenuation is crucial since it enables a more precise characterization of the ice and basal conditions. Although such problem has been often addressed in the literature, the assessment of the ice attenuation is subject to several hypotheses and uncertainties, resulting in a wide range of possible interpretations of the properties of the ice. In this paper, we propose a method for constraining the ice attenuation profiles in the vicinity of an ice core by jointly using coincident radar sounder (RS) data (radargrams) and dielectric profile (DEP) data. Radargrams contain measurements of radar reflected power from ice subsurface dielectric discontinuities (layers) on wide areas. DEP data cointain ice dielectric permittivity measurements collected at an ice core. The method relies on the detection of ice layers in the radargrams, the estimation of their depth and reflectivity from the DEP data, and the use of the radar equation for the estimation of ice attenuation through the whole ice column and locally at each layer position. The method has been applied to RS and DEP data acquired at the NEEM core site in Greenland. Experimental results confirm the effectiveness of the proposed method.
\end{abstract}

Keywords: ice sheet, radar sounder, ice dielectric profile, ice attenuation

\section{INTRODUCTION AND BACKGROUND}

The importance of better understanding the ice attenuation has been often highlighted by the scientific community, e.g.,. ${ }^{1} .^{2}$ To address this challenge, most of the available studies rely on the analysis of radargrams acquired by radar sounder (RS) instruments operated at the ice sheets. Radar sounders are non-intrusive instruments that transmit low-frequency electromagnetic waves vertically towards the ice. Radargrams are 2D matrices containing measurements of the power reflected by the ice subsurface dielectric discontinuities on wide areas, as a function of wave travel time in the vertical direction and as a function of platform location in the horizontal direction. Thus, radargrams show the surface and subsurface features (layers of ice and underlaying bedrock). The ice attenuation can be estimated by analyzing the radar power vertical profiles in the radargrams. It is well known that the received power as a function of depth depends on multiple factors affecting the wave transmitted power, such as: geometric spreading losses in air and ice; reflection and scattering losses at the ice surface, internal layers and bedrock; and attenuation in the ice subsurface. ${ }^{3}$ The geometric losses depend on the distance between sensor and target, the reflection and scattering losses depend on the interface dielectric contrast and roughness, and the ice attenuation is due to ice impurity and conductivity, which is a function of instrument working frequency and ice temperature. ${ }^{4}$

Most methods for inferring the ice attenuation employ bedrock-driven approaches (BDA), e.g.,, ${ }^{1 .}$ Such methods rely on the relationship between the ice thickness and the power returned from the bedrock, and assume constant reflectivity of the bedrock along the radargram in order to estimate the ice attenuation rate. This assumption is the main weakness of such methods, since it is unlikely to hold on wide areas.

Other methods for the estimation of ice attenuation are based on layer-driven approaches (LDA) e.g., ${ }^{2}{ }^{6}{ }^{6}$ This implies the use of information about the layers visible in the radargram rather than information about the bedrock reflected power. The ice internal layers have been generated over millennia by snow accumulation on 
the underlying bedrock, alternated by depositions of impurities from volcanic explosions and ice flow dynamics, therefore they have an isochronous character. ${ }^{7}$ The main advantage of the LDA approaches is that they do not rely on the bedrock reflections to estimate the ice attenuation rate, since the reflections of the bedrock are more complex and proven to be spatially variable, e.g.,, 8.9 The LDA methods rely on the isochronous character of the ice layers. Recently, a method based on LDA has been presented in. ${ }^{2}$ The method uses a linear regression technique to estimate the depth-averaged attenuation rate and relies on the assumption that the internal layers highlighted in radargrams are specular and that the reflectivity of the layers is uniform in the range direction. The LDA and the assumption of uniform vertical reflectivity have been also used in ${ }^{6}$ to study the spatial uniformity of the attenuation.

In this paper, we propose a method for constraining the ice attenuation profiles in the vicinity of an ice core by jointly using coincident RS data and measurements of the ice dielectric permittivity (DEP) collected at an ice core. The method applies to the RS data the LDA, in which, unlike the above-mentioned works, the layer reflectivities are estimated from the available DEP data. Our choice of jointly using RS and DEP data combined with the LDA method is motivated by the observation ${ }^{10}$ that an important consequence of the isochronous character of the ice layers is the fact that it allows the findings at any given drill site to be extrapolated over a region covered by an RS survey that passes through the drill site. Moreover, our choice of estimating the layer reflectivities from the DEP is due to the fact that, as noted in, ${ }^{2}$ the assumption of uniform vertical reflectivity does not hold along the entire vertical profile since there are several radar bright reflections due to higher variations in layer reflectivity. Furthermore, as observed in, ${ }^{11}$ radar-derived attenuation rates to several reflectors could constrain the ice attenuation rate profile and provide additional tests for the attenuation models.

The method has been applied to RS data acquired by the MultiChannel Coherent Radar Depth Sounder (MCoRDS) instrument ${ }^{12}$ and DEP data acquired at the NEEM ice core site $^{13}$ in Greenland.

The rest of the paper is organized as follows. Sec. 2 provides a detailed description of the proposed method. Experimental results are given in Sec. 3. Finally, Sec. 4 draws the conclusions of this work and provides ideas for future developments.

\section{PROPOSED METHOD FOR ICE ATTENUATION ESTIMATION}

\subsection{Problem formulation and architecture of the proposed method}

A radargram is a $2 \mathrm{D}$ matrix of dimension $\left[n_{S}\right.$ samples/rows $\times n_{T}$ traces/columns] that contains the power reflected by interfaces in the ice subsurface. Such measurements are recorded as a function of two-way travel time $(2 \mathrm{WTT}) \Lambda$ to the interface (or sample $i=\left[1 . . n_{S}\right]$ ) in the range direction, and as a function of platform position (or trace $t=\left[1 . . n_{T}\right]$ ) in the along-track direction. An ice dielectric profile (DEP) contains measurements of the dielectric permittivity of the ice along the ice column. Our technique jointly uses RS and DEP data, which we assume coincident at the ice core position $t=t^{c}$.

The proposed method is a trace-based technique which relies on the use of the radar equation at each trace and assumes that the ice internal layers visible in the radargram are dominated by specular reflections. According to the radar equation for an airborne mounted nadir-looking ice sounder (commonly operated at the ice sheets), the power $P_{k}$ measured by the RS receiver at trace $t$ from a specular layer $k$ located at a depth $Z_{k}$ is given by:

$$
P_{k}=P_{t x} \cdot\left(\frac{\lambda}{4 \pi}\right)^{2} \cdot \frac{G^{2}}{\left[2\left(H+\frac{Z_{k}}{\sqrt{\varepsilon_{k}}}\right)\right]^{2}} \cdot L_{k}^{2} \cdot \Upsilon_{s}^{2} \cdot \Gamma_{k},
$$

where $P_{t x}$ is the transmitted pulse power, $\lambda$ is the wavelength, $H$ is the height of the RS platform with respect to the surface, $G$ is the gain of the antenna, $\varepsilon_{k}$ is the dielectric permittivity at layer $k$ and $L_{k}$ is the one way power loss due to the ice attenuation until layer $k . \Gamma_{k}$ is the reflectivity of the layer $k$, and is given by the dielectric contrast at the layer interface. $\Upsilon_{s}$ is the transmission coefficient of the surface and is given by:

$$
\Upsilon_{s}=1-\rho_{s} \Gamma_{s}
$$

where $\rho_{s}$ is the reflection reduction due to surface roughness and $\Gamma_{s}=0.029$ (which is equivalent to $-15.31 \mathrm{~dB}$ ) is the reflectivity of the surface, i.e., the interface between air and snow. It is important to note that by using (1) 


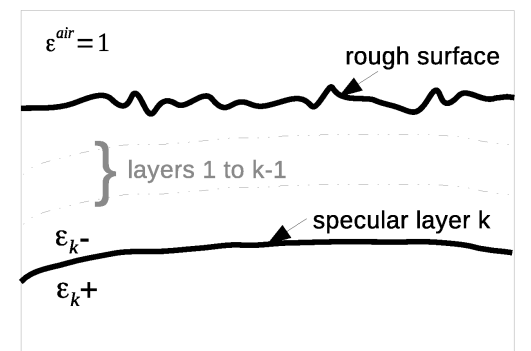

Figure 1. Two interface model of the ice sheet.

we implicitly assume an ice sheet simplified model composed of two interfaces, i.e., the ice surface and the layer $k$, as depicted in Fig. 1. The intermediate $k-1$ internal layers are assumed to have a transmission coefficient close to 1, because of their low reflectivity. For this reason and for the sake of clarity, the product of transmission coefficients until layer $k$ is omitted in (1).

By expressing the received power from layer $k$ in relation to the received power from the surface, the derived power ratio is independent on the parameters of the $\mathrm{RS}$, and is given by:

$$
\frac{P_{k}}{P_{s}}=\frac{\left(1-\rho_{s} \Gamma_{s}\right)^{2} \Gamma_{k}}{\rho_{s} \Gamma_{s}} \cdot\left(\frac{H}{H+\frac{Z_{k}}{\sqrt{\varepsilon_{k}}}}\right)^{2} \cdot L_{k}^{2},
$$

where $P_{s}$ is the received power from the surface, given by:

$$
P_{s}=P_{t x} \cdot\left(\frac{\lambda}{4 \pi}\right)^{2} \cdot \frac{G^{2}}{(2 H)^{2}} \cdot \rho_{s} \Gamma_{s} .
$$

Moreover, by inverting (3) one can derive $L_{k}$ :

$$
L_{k}=\left[\frac{P_{s}}{P_{k}} \cdot \frac{\rho_{s} \Gamma_{s}}{\left(1-\rho_{s} \Gamma_{s}\right)^{2} \Gamma_{k}} \cdot\left(\frac{H+\frac{Z_{k}}{\sqrt{\varepsilon_{k}}}}{H}\right)^{2}\right]^{1 / 2} .
$$

It is worth noting that $L_{k}$ can be estimated for all layers $k$ and for all traces $t$ with (5) as a discrete function of layer depths $Z_{k}$ and other properties of the surface and layers, i.e., surface reflectivity, power and roughness reduction coefficient, and layer power and reflectivities, which are all unknown quantities, to be estimated from the data.

In this work we aim to estimate the ice attenuation profiles for all traces of the radargram as a function of layer depth through the whole ice column and the attenuation at the bedrock, starting from (5). To this aim, we propose a method composed of 5 blocks. The block scheme of the technique is depicted in Fig. 2. In particular, the first block performs the surface and bedrock detection, and surface roughness estimation from the radargram, in order to provide the required $P_{s}$ and $\rho_{s}$ at all positions $t$ of the platform. The second block performs the layer detection and provides the $2 \mathrm{WTT} \Lambda_{k}$ and the power $P_{k}$ of $K$ specular layers that are passing through the ice core position $t^{c}$. The depth of the layers $Z_{k}$ at the position $t^{c}$ of the DEP and the reflectivity of the layers $\Gamma_{k}$ are estimated in the third block of the technique by jointly using the RS and DEP data. At this point all the unknowns, i.e., $P_{s}, P_{k}, \rho_{s}, \Gamma_{s}, \Gamma_{k}, Z_{k}, \varepsilon_{k}$ in (5) are estimated, and thus the discrete ice attenuation $L_{k}$ at the depth of each layer $k$ at the DEP position $t^{c}$ can be calculated. Moreover, the considered two interface model of the ice sheet and the assumption that the layers are dominated by specular reflections (i.e., the reflectivity $\Gamma_{k}$ of each layer is constant along the layer $k$ ) allows us to estimate the layer depth and consequently the corresponding ice attenuation $L_{k}$ for all traces $t$ where they have been detected. We perform this in the fourth block of the technique. Finally, we fit a continuous function to the discrete ice attenuation at the layer depths and extrapolate it in order to estimate the ice attenuation at the depth of the bedrock.

In the following subsections, a detailed description of each of the processing steps of the method is given. 


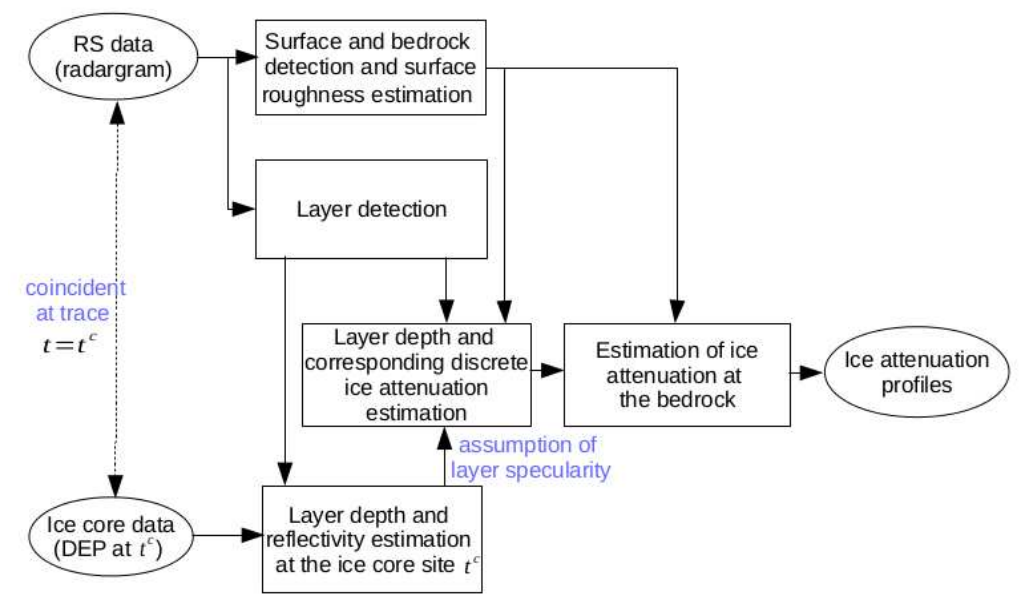

Figure 2. Block scheme of the proposed technique for the ice power loss estimation.

\subsection{Step 1: Surface and bedrock detection and estimation of reflection reduction due to surface roughness}

The objective of this step is the detection of surface and bedrock positions, and the estimation of the surface power $P_{s}$ and of the reflection reduction $\rho_{s}$, for the entire radargram.

The surface and bedrock positions can be traced manually or according to automatic techniques, e.g.,. ${ }^{14}$ Once the surface position and the related power $P_{s}$ are extracted from the radargram, we use them to estimate the surface roughness parameters, i.e., the root mean square height RMSH $\sigma_{h}$ and the correlation length $L C$, which are in turn used to estimate $\rho_{s}$. In particular, $\sigma_{h}$ represents the vertical displacement of the surface with respect to its mean plane, whereas $L C$ is the horizontal length over which the samples are correlated. These quantities are estimated with the method proposed in. ${ }^{15}$

\subsection{Step 2: Layer detection}

The objective of this step is the detection of the well-defined ice internal layers and the computation of their power $P_{k}$ for all the traces $t$ of the radargram. To this aim we use the method proposed in. ${ }^{16}$ The method uses the phase information measured by the RS and detects the layers based on the analysis of the Doppler spectrum (for further details on the method the reader is referred to ${ }^{16}$ ). We apply this method and detect the power of the layers $P_{k}$ and their position as a function of $2 \mathrm{WTT} \Lambda_{k}$, along the entire radargram. Afterwards, we select only those $K$ layers that are passing through the ice core site. Under the assumption of layer specularity, which is verified according to the method proposed in, ${ }^{16}$ this enables the findings at the ice core (see Sec. 2.4) to be extrapolated over the radargram by following the layers (see Sec. 2.5).

\subsection{Step 3: Layer depth and reflectivity estimation at the ice core site}

The aim of this step is the computation of the layer depths and reflectivities. Initially, we achieve this at the ice core position $t=t^{c}$ by jointly using the DEP data with the information extracted from the RS data. Note that the DEP data are available in the depth domain, i.e., a measurement of dielectric permittivity is taken every $\approx \delta_{z}^{D E P}[\mathrm{~m}]$, where $\delta_{z}^{D E P}$ is the vertical resolution of the DEP profile. On the contrary, the $K$ layers at the ice core are detected in the time domain, i.e., as a function of 2 WTT $\Lambda_{k}$. Thus, in order to match the layers visible in the radargram at $t=t^{c}$ to their position in the DEP profile, a conversion from time to depth should be performed. Therefore, in order to compute the layer depth and reflectivity at the ice core site we perform three main operations: i) conversion of the DEP depth to time domain and matching of $\Lambda_{k}$ extracted from the radargram at $t=t^{c}$, ii) extraction of the corresponding dielectric permittivity for the computation of $\Gamma_{k}$, and iii) back conversion from time to depth domain for the estimation of the layer depth $Z_{k}$. This sequence of operations is done for all layers $k=[1 . . K]$ detected in the radargram at the DEP ice core site $t^{c}$. 
It is worth mentioning an important aspect regarding the estimation of the layer reflectivity. Generally, the depth resolution of the RS data is much coarser than that of the DEP data. Thus, several dielectric interfaces (elementary scatterers) in the DEP can contribute to the reflectivity effectively seen by the RS in a resolution cell at the position of the layer in the radargram. Since at our knowledge the estimation of the effective layer reflectivity has been only marginally addressed in the literature, e.g., ${ }^{17}$ we are studing two potential approaches to the estimation of the effective reflectivity:

- $\Gamma_{k}^{M}$ computed as the mean value (MVA) of the reflectivity contributions coming from all interfaces in the DEP that are within the RS range resolution cell at $\Lambda_{k}, \forall k=[1 . . K]$, where each interface contributes with a reflectivity given by the Fresnel law, $\Gamma_{i}^{F}=\frac{\sqrt{\varepsilon_{i}}-\sqrt{\varepsilon_{i-1}}}{\sqrt{\varepsilon_{i}}+\sqrt{\varepsilon_{i-1}}}$, where $i$ is the considered interface in the DEP.

- $\Gamma_{k}^{C}$ computed as the coherent sum (CSA) of the reflectivity contributions coming from all interfaces in the DEP that are within the RS range resolution cell at $\Lambda_{k}, \forall k=[1 . . K]$. In this case, each interface contributes to the final effective reflectivity with an amount $\Gamma_{i}^{F} e^{\left(j \phi_{i}\right)}$, where $\phi_{i}=4 \pi\left(H / \lambda_{\text {air }}+z_{i} / \lambda_{i c e}\right), z_{i}$ is the depth of the considered interface in the DEP profile and $\lambda_{i c e}$ the wavelength in the ice. This is a similar concept with the speckle, which is the coherent sum of the contributions from all elementary scatterers present in a radar resolution cell.

It is also worth to note that the layer depths are typically computed by using the range to target equation, in which a constant dielectric permittivity of ice $\varepsilon^{i c e}=3.15$ is assumed. However, with this approximation the layer depths are under- or over-estimated, leading to an incorrect layer depth matching in the DEP and consequently to an incorrect layer reflectivity estimation. On the contrary, the availability of the DEP allows us to improve the estimation of the layer depth and reflectivity, as previously explained.

\subsection{Step 4: Layer depth and ice attenuation profile estimation}

The objective of this step is twofold: i) the estimation of the depth of the selected layers on the whole radargram, and ii) the estimation of the ice attenuation profiles as a discrete function of layer depth along the radargram.

The computation of the layer depths in the whole radargram is done similarly to the estimation of the layer depths at the DEP site (see Sec. 2.4). The only difference is that for all traces $t \neq t^{c}$ there is no information on the dielectric profile. Therefore, in order to estimate the layer depths $Z_{k}$, we assume that between the surface and the layer $k$ at traces $t \neq t^{c}$, the dielectric profile is a scaled version of the DEP between the surface and the layer $k$ at the ice core $t^{c}$. It is worth noting that this is a consequence of the two interface ice sheet model that we are considering (see Sec. 2.1). For a multilayer model, one can assume that between each two adjacent layers $k$ and $k+1$ at traces $t \neq t^{c}$, the dielectric profile is a scaled version of the DEP between $k$ and $k+1$ at the ice core $t^{c}$. Furthermore, under the assumption that the layers are characterized by specular reflections, $\Gamma_{k}$ computed at the DEP site $t^{c}$ (see Sec. 2.4) is constant along the layer, therefore known for all the selected layers on the whole radargram.

At this stage, the ice attenuation profile as a discrete function of layer depth $L_{k}$ can be computed for the whole radargram by using (5), in which all the unknowns have been estimated as previously explained.

\subsection{Step 5: Estimation of ice attenuation at the bedrock}

The final aim of the proposed technique is the estimation of the ice attenuation at the bedrock, starting from the estimated discrete ice power losses $L_{k}$ at the layer depths. To this aim, we propose the following trace-based approach. For each trace $t$, we regard the estimated discrete $L_{k}$ as samples in the depth-power domain, to which we fit a continuous function. By extrapolating the function we estimate the ice attenuation at the depth of the bedrock. Regarding the fit, it is worth noting the impact of the fitting function on the estimation given the number of layers and their distribution along the trace. In order to ensure a reliable and meaningful fit, a sufficient number of samples should be available with an ideal uniform distribution with depth. Thus, the smaller the number of layers and the more complex the distribution of $L_{k}$ with depth, the worse the fitting performances. Regarding the fitting function, we are using an exponential model of ice attenuation, i.e., a linear model in the log-power domain. ${ }^{1}$ 


\section{EXPERIMENTAL RESULTS}

We applied the proposed technique to RS data acquired by the MCoRDS instrument ${ }^{12}$ over a distance of $90 \mathrm{~km}$ and DEP data acquired at the NEEM ice core site $^{13}$ in Greenland. The RS data are acquired at low altitude, i.e., $H=480 \mathrm{~m}$ above the surface, at central frequency $f_{c}=195 \mathrm{MHz}$ and bandwidth $B_{w}=30 \mathrm{MHz}$. The data are range compressed with a weighting factor 1.53, and the range resolution in ice is $\delta_{z}^{R S}=4.3 \mathrm{~m}$. The DEP data are acquired with a range resolution $\delta_{z}^{D E P} \approx 5 \mathrm{~mm}$. The proposed technique assumes RS and DEP data coincident at the ice core position. However, the investigated RS and DEP data are not perfectly coincident; the closest trace of the radargram to the ice core $t \approx t^{c}$ is at a distance of $1.6 \mathrm{~km}$.

The investigated RS data are shown in Fig. 3(a), whereas the NEEM DEP profile is shown in Fig. 3(b). The closest trace to NEEM $t \approx t^{c}$ is depicted with a vertical red line in Fig. 3(a), whereas the top, bottom and intermediate horizontal lines in Fig. 3(a) represent the output of the detection of the surface, bedrock and of the specular layers that are passing through the NEEM site, respectively.

Fig. 3(c) reports the depth offset (see Sec. 2.4), obtained between the corrected depth (calculated at the core site by considering the available DEP measurements) and the approximated depth (calculated with the typically used constant $\varepsilon_{i c e}=3.15$ ), against the corrected depth. As it can be seen, the offset at the depth of the detected layers is in the range between $8 \mathrm{~m}$ and $13 \mathrm{~m}$, which is in the order of 2-3 times the RS range resolution cell. The effective reflectivity of the layers $\Gamma_{k}^{C}$ and $\Gamma_{k}^{M}$, obtained with the two investigated methods CSA and MVA, respectively, see Sec. 2.4) at the corrected depth of the layers is depicted in Fig. 3(d). As it can be seen, the approach based on the coherent sum provides much higher reflectivities (average value of $\Gamma^{C}=-34.6 \mathrm{~dB}$ ) than the approach based on the mean value (average value of $\Gamma^{M}=-72.59 \mathrm{~dB}$ ). We use the values of the reflectivity $\Gamma_{k}^{C}$ and estimate the discrete attenuation at the layer depths as explained in Sec. 2.5. Then, by linearly fitting these discrete attenuation values and by extrapolating the function to the bedrock, we obtain attenuation values at the bedrock in the range of $-46 \mathrm{~dB}$ to $-30 \mathrm{~dB}$, for bedrock depths in the range from $2350 \mathrm{~m}$ to $2700 \mathrm{~m}$, see Fig. $3(\mathrm{e})$. These values provide an average ice attenuation rate of $-14.32 \pm 1.34 \mathrm{~dB} / \mathrm{km}$, see Fig. $3(\mathrm{e})$, which are within the range of uncertainty of previous estimates at NEEM. ${ }^{2}$ Two examples of attenuation at the discrete layer depths obtained by using $\Gamma_{k}^{C}$ at are given in Fig. $3(\mathrm{~g})$ and $3(\mathrm{~h})$. The analyzed traces are highlighted with vertical orange and cyan lines in Fig. 3(a). As is can be seen in Fig. 3(g) and Fig. 3(h), the attenuation profile shows an overall decreasing trend. However, it is not monotonically decreasing as expected. This can be due to possible surface clutter or layer slope effects that have not been taken into account in this work. On the other hand, by using the values of $\Gamma_{k}^{M}$ we frequently obtain (for several layers, for several traces) positive attenuation values at the discrete layer depths and attenuation rates of few $\mathrm{dB} / \mathrm{km}$, which are not confirmed by previous studies and also physically not explicable. Thus, we derive that between the two approaches for the estimation of the effective reflectivity of the layers seen in the radargram, the CSA is more suitable than MVA.

As it has been shown, besides providing a validated ice attenuation rate, the method relying on CSA also offers the possibility to improve the estimation of the depth of the layers and to infer the ice attenuation locally at each layer depth. Moreover, since the method estimates the reflectivity of the layers based on real DEP measurements, it also provides an approach to constraining the ice attenuation profile at the detected layer depths, contrary to other methods that assume constant vertical layer reflectivity.

\section{CONCLUSION}

In this paper we described a method for constraining the ice attenuation profile in the vicinity of an ice core. The method jointly uses coincident RS and ice core DEP data, which is the novel contribution of this work, and it is based on the use of the radar equation. In particular, the DEP measurements are used to estimate the depths and the reflectivities of the specular layers visible in the radargram, which are passing through the ice core. We studied two approaches for the estimation of the effective reflectivity of the layers seen in the RS data: an approach based on the mean value (MVA) of the contributions coming from all interfaces in the DEP that are within the RS range resolution cell at the layer depths, and an approach based on the coherent sum (CSA) of the contribution inside the radar range resolution cell. Then, we inverted the radar equation in order to estimate the discrete ice attenuation at the detected layer depths. The attenuation at the bedrock has been estimated by fitting and extrapolating a linear function (in the log-power domain) to the estimated discrete ice attenuation. 


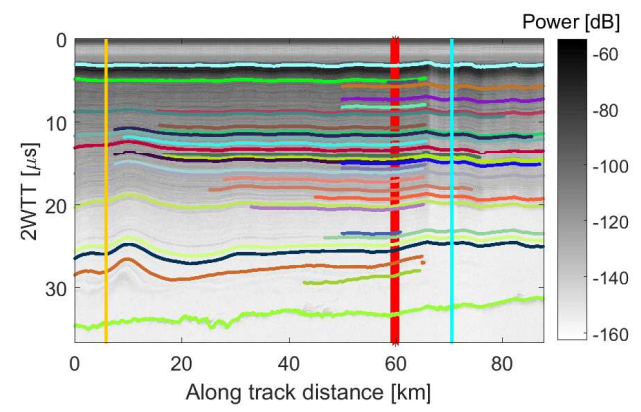

(a)

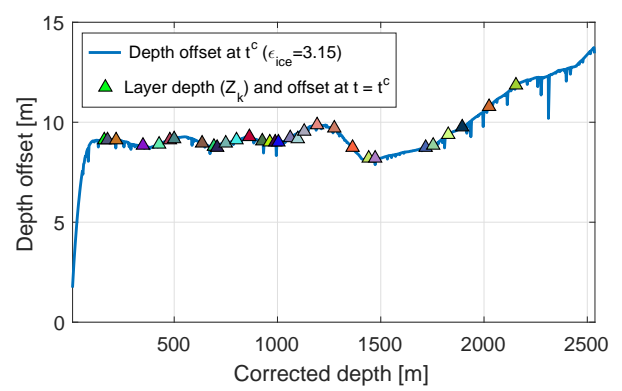

(c)

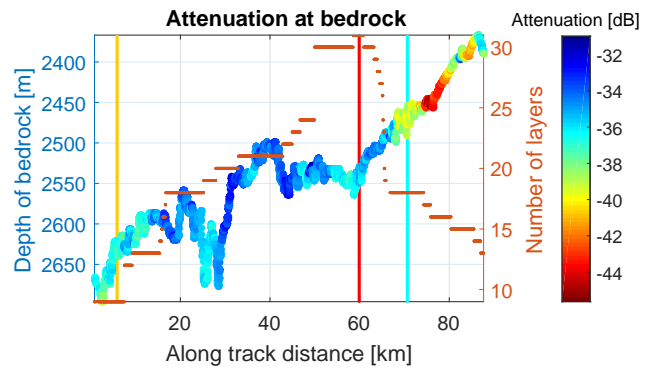

(e)

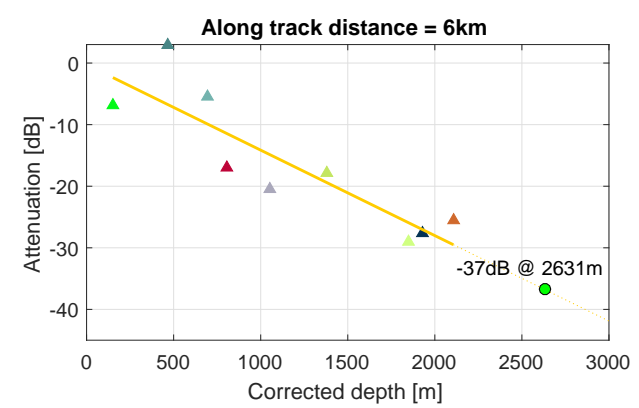

(g)

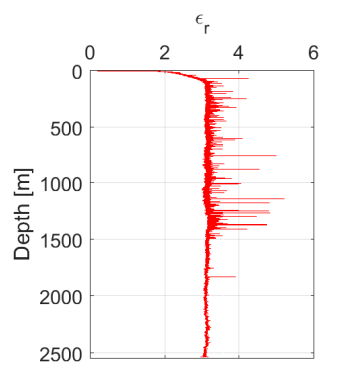

(b)

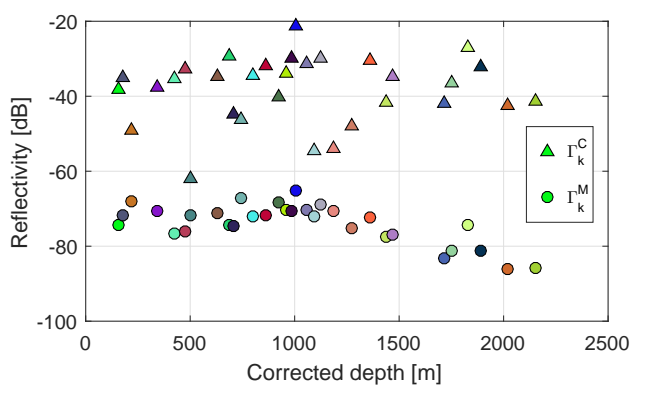

(d)

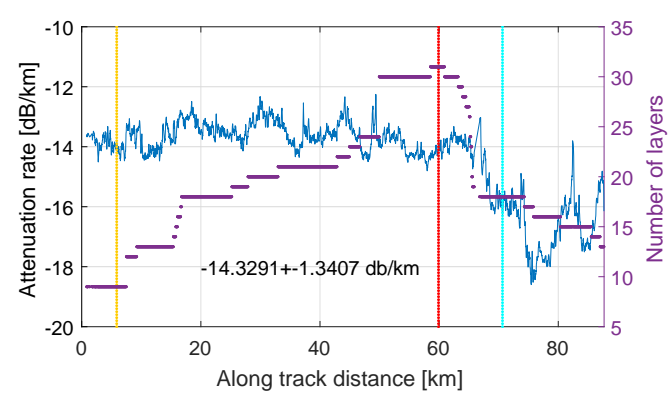

(f)

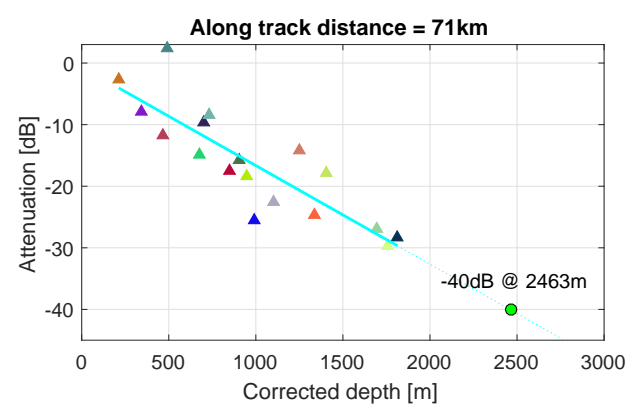

(h)

Figure 3. (a) Input RS dataset, the red vertical line represents the NEEM ice core position, the horizontal lines represent the detected surface, bedrock and specular layers passing through NEEM; (b) Input DEP data at NEEM; (c) Corrected depth versus offset depth calculated at NEEM; (d) Estimated effective reflectivity of the layers at NEEM computed with the two studied approaches, i.e., based on the mean value and on the coherent sum of the DEP interfaces in the radar resolution cell; (e) Estimated depth and ice attenuation at the bedrock; (f) Estimated ice attenuation rate; (g), (h) Examples of ice attenuation profiles at the traces highlighted in Fig. 3(a) with orange and cyan vertical lines, respectively. 
We applied the method to DEP data and to a $90 \mathrm{~km}$ RS dataset acquired by the MCoRDS instrument at the NEEM ice core site in Greenland. With CSA we obtained an ice attenuation rate of $\approx-14 \mathrm{~dB} / \mathrm{km}$, which is within the uncertainty range of previous estimates. On the contrary, the MVA provided attenuation rates that are physically not explicable. Thus, we derived that between the two approaches for the estimation of the effective reflectivity of the layers seen in the radargram, the CSA is more suitable than MVA. Besides providing a validated attenuation rate, the method relying on CSA also provides an approach to constraining the ice attenuation profile at the detected layer depths which is likely more precise than assuming constant vertical layer reflectivity.

As future work we aim to study the effect of the DEP resolution on the estimated effective layer reflectivity. Moreover, we plan to model possible surface clutter and layer slope effects and to study the sensitivity of the method to the number of detected layers and their distribution along the vertical profile.

\section{REFERENCES}

[1] Jacobel, R., Welch, B., Osterhouse, D., Pettersson, R., and MacGregor, J., "Spatial variation of radarderived basal conditions on Kamb Ice Stream, West Antarctica," Annals of Glaciology 50(51), 10-16 (2009).

[2] MacGregor, J., Li, J., Paden, J., Catania, G., Clow, G., Fahnestock, M., Gogineni, S., Grimm, R., Morlighem, M., Nandi, S., Seroussi, H., and Stillman, D., "Radar attenuation and temperature within the Greenland Ice Sheet," Journal of Geophysical Research: Earth Surface 120(6), 983-1008 (2015).

[3] Bogorodsky, V., Bentley, C., and Gudmandsen, P., [Radioglaciology] (1984).

[4] Matzler, C. and Wegmuller, U., "Dielectric properties of freshwater ice at microwave frequencies," Journal of Physics D: Applied Physics 20(12), 1623 (1987).

[5] Gades, A., Raymond, C., Conway, H., and Jacobel, R., "Bed properties of Siple Dome and adjacent ice streams, West Antarctica, inferred from radio-echo sounding measurements," Journal of Glaciology 46(152), 88-94 (2000).

[6] Matsuoka, K., Morse, D., and Raymond, C., "Estimating englacial radar attenuation using depth profiles of the returned power, central West Antarctica," Journal of Geophysical Research: Earth Surface 115(F2) (2010).

[7] Robin, G., Evans, S., and Bailey, J., "Interpretation of radio echo sounding in polar ice sheets," Philosophical Transactions of the Royal Society of London. Series A, Mathematical and Physical Sciences 265(1166), 437505 (1969).

[8] Winebrenner, D., Smith, B., Catania, G., Conway, H., and Raymond, C., "Radio-frequency attenuation beneath Siple dome, West Antarctica, from wide-angle and profiling radar observations," Annals of Glaciology 37(1), 226-232 (2003).

[9] Schroeder, D. Grima, C. and Blankenship, D., "Evidence for variable grounding-zone and shear-margin basal conditions across Thwaites Glacier, West Antarctica," Geophysics 81(1), WA35-WA43 (2015).

[10] Miners, W., Wolff, E., Moore, J., Jacobel, R., and Hempel, L., "Modeling the radio echo reflections inside the ice sheet at Summit, Greenland," Journal of Geophysical Research: Solid Earth 107(B8) (2002).

[11] MacGregor, J., Winebrenner, D., Conway, H., Matsuoka, K., Mayewski, P., and Clow, G., "Modeling englacial radar attenuation at Siple Dome, West Antarctica, using ice chemistry and temperature data," Journal of Geophysical Research: Earth Surface 112(F3) (2007).

[12] Rodriguez-Morales, F., Gogineni, S., Leuschen, C., Paden, J., Li, J., Lewis, C., Panzer, B., GomezGarcia Alvestegui, D., Patel, A., Byers, K., Crowe, R., Player, K., Hale, R., Arnold, E., Smith, L., Gifford, C., Braaten, D., and Panton, C., "Advanced multifrequency radar instrumentation for polar research," IEEE Transactions on Geoscience and Remote Sensing 52(5), 2824-2842 (2014).

[13] Rasmussen et al., S., "A first chronology for the North Greenland Eemian Ice Drilling (NEEM) ice core," Climate of the Past 9(6), 2713-2730 (2013).

[14] Ilisei, A.-M. and Bruzzone, L., "A system for the automatic classification of ice sheet subsurface targets in radar sounder data," IEEE Transactions on Geoscience and Remote Sensing 53(6), 3260-3277 (2015).

[15] Neal, C., "Radio echo determination of basal roughness characteristics on the Ross Ice Shelf," Annals of Glaciology 3, 216-221 (1982). 
[16] MacGregor, J., Fahnestock, M., Catania, G., Paden, J., Gogineni, S., Young, S. K., Rybarski, S., Mabrey, A., Wagman, B., and Morlighem, M., "Radiostratigraphy and age structure of the Greenland Ice Sheet," Journal of Geophysical Research: Earth Surface 120(2), 212-241 (2015).

[17] Harrison, C., "Radio echo sounding of horizontal layers in ice," Journal of Glaciology 12, 383-397 (1973). 